\title{
Moving toward NextGenetic Counseling
}

\author{
Myra I. Roche, MS ${ }^{1}$
}

Many figurative hands have been wrung over how to reconcile the collision of an irresistible force with a seemingly immovable object. The force: the burgeoning amount of genetic information obtainable by genome-wide tests. The obstacle: the practical constraints imposed by the limited number of providers qualified to deliver it in clinically meaningful and ethical ways. This is not a new problem, and solutions have been proposed. One idea, popular for a while, was to expand our ranks to include other health professionals, effectively outsourcing the problem. But after several attempts to develop and provide training for "fill in your favorite health professional here", and to train additional genetic counselors and medical geneticists, our capacity has not appreciably swelled. Even so, this blip hardly registers in comparison with the imminent genomic tsunami hovering off our shores, threatening to drown us all in a downpour of sequence data.

If the finite number of qualified professionals is largely static yet the number of people waiting to be counseled is increasing exponentially, the next logical target would be paring down the length of the session. Ignoring the option of simply talking faster, one choice would be to present less information. This strategy runs exactly counter to the prevailing winds of the aforementioned genomic tsunami. Lacking any better ideas, we typically rely on auxiliary communication methods that supplement rather than supplant us. While the spectrum includes group counseling, teleconferencing, and computer modules, the most commonly used device is some variation of that old standby, the patient pamphlet.

As a ubiquitous, side-car companion to the process of patient education, the pamphlet has shown remarkable resilience. When offered after a session, a well-designed pamphlet can be used to review and enhance information. Given before an appointment, it has the potential to prepare the necessary groundwork. Using established design techniques, a pamphlet (paper or virtual) can successfully introduce genetic content and, equally as important, can prime people for the potentially complex decisions we ask them to make. ${ }^{1}$ It is misguided, however, to expect ancillary sources to quell patients' desires for information, thus enabling a truncated visit. In fact, quite the opposite occurs. Easy access to genetic information has stimulated a voracious appetite that devours vast amounts of Internet information, much of which remains largely indigestible because it is too complex, irrelevant, and, often, too generic for general consumption. ${ }^{2}$

Resculpting the session content should be put on the surgical table, but I suspect that my 3,000 (give or take a few) medical genetics colleagues who provide clinical services are already operating pretty close to the bone forced by the twin necessities; time and money. Lofty descriptions of the components of a "traditional genetic counseling session" found in journal articles have few real-world counterparts inside most medical genetics clinics unless they exist in a financially protected environment. Those of us in the unprotected trenches are reminded of this fantasy world whenever a new student steps into ours and tries to follow that outdated playbook. Still, we could take a stab at it.

Let's admit it though, even a svelte genetic counseling session takes a lot of time-there's a lot to do. Here's a list: convey medical and genetic information, translate implications, communicate in a way to optimize understanding, aid psychological adjustment, and ensure that decision-making is informed. It's a pretty tall order for a profession whose very name evokes confusion. Like George Carlin's riff on "jumbo shrimp”, those two words-genetic and counseling-seem almost paradoxical. Professionally, genetic counseling is a chimerical offspring of estranged parents each espousing a distinct methodological view of practice; the directive, expert-driven model of traditional medicine vs. a Rogerian, client-centered, psychotherapeutic approach. $^{3}$

This hybrid blend of education and counseling challenges efforts to develop comprehensive outcome measures. In contrast to the even more poorly reimbursed counseling half, the education half is really our only chance to measure, replicate, and outsource. The most commonly studied outcomes are focused there, on those things we can best count; knowledge acquisition, risk comprehension, and reproductive decisions. These have been measured over and over and over again. Squishier concepts like psychological distress and patient satisfaction often get shorter shrift, in part because we don't quite know what to do with the results. Valid outcome measures depend upon correctly choosing what to measure, how to do it, and, just as importantly, on whom.

In their search for a leaner way to disclose apolipoprotein E genotypes and provide risk education for Alzheimer disease, ${ }^{4}$

${ }^{1}$ Pediatrics and Genetics, University of North Carolina-Chapel Hill, Chapel Hill, North Carolina, USA. Correspondence: Myra I. Roche (myra_roche@med.unc.edu) 
Roberts and his REVEAL colleagues begin by aiming their knife at these same educational outcomes, knowledge and risk comprehension, as they whack away at the bloated genetic counseling session. The study sought to identify if there were "significant differences that would argue against using a condensed protocol" vs. the not-so-subtly-named, extended protocol. We should be clear: the success of the intervention is not judged by whether the two groups show equivalent knowledge, but rather, the experiment is labeled a failure only if those in the experimental group show significantly worse knowledge.

The authors were quite right to pay due diligence by being cautious about pre-empting an established protocol for returning results for a condition like Alzheimer disease. And I agree that their study population is not really comparable with candidates for predictive Huntington disease testing, for whom the "extended protocol" was initially designed. Their conclusion, that the two groups did not differ statistically in their recall of their apolipoprotein E genotype or their lifetime risk, was meant to allow them to pry the gate open, just a bit, paving the way for similar models of risk assessments for common, complex diseases. But context is sorely needed here and, as it turns out, the whole class got an " $\mathrm{A}$ " on the test because everyone was, like the children in Lake Wobegon, above average. The characteristics of the population assayed are intimately related to the degree to which the conclusions of the study can be generalized. This population was highly skewed and hardly representative of the general population with common, complex diseases. To their credit, the authors admit that their participants were highly educated, with high numeracy scores, intimately familiar with the condition, clinically stable, self-referred, and highly motivated. While I won't quibble here about confounders such as the face validity of the items used to test knowledge, the conclusions reached from this population simply cannot be extrapolated to a broader one, thereby seriously compromising the goal: to show the efficacy of briefer models of genetic risk assessment to people with common, complex diseases.

Still, what about those 43 minutes per person saved by nudging them a little closer to using a self-checkout line for return of their test results? Although not for everyone, there may be rational ways to triage people into different lines so that those comfortable with and capable of using a nonhuman adjuvant are not forced to stand in the same lines with those who aren't and can't. ${ }^{5}$ The direct to consumer market has already proven this principle. Otherwise, patient education and genetic counseling face the danger of being labeled the last stubborn bottleneck in a high-throughput process. Shouldn't we turn around to face the growing lines of data and patients piling up behind us?
Genetic counseling has evolved like a population of organisms, by simply piling on new functions as they arise without jettisoning any of the antiquated components. But the true weight of the ballast is finally catching up with us and, in the face of the data downpour, needs to be reckoned with.

Let's start by sharpening our knives. But before cutting we need to determine, empirically, which parts of the genetic counseling process are comparable with the exomes, the vital coding sequences of the communication process that must be preserved and maintained, and, which parts are like the introns, parts that can, and must, be excised and thrown overboard? Could alternative splicing be done, tailoring the messages to retain their highly personalized quality yet packaged for more efficient delivery than by a face-to-face encounter? Could we create more relevant content, while minimizing adverse outcomes, if we understood how differences in information seeking behaviors translated to decisions to seek some kinds of incidental results identified by genomic analyses? Studying which characteristics trigger some people to learn and some to shun different kinds of results could improve the informed consent process. And by capitalizing on the principles of successful communication models such as social media, new ways may emerge allowing us to connect to diverse groups more efficiently, yet still humanely.

It is ironic that, in this era of "personalized genomic medicine," genetic counseling, a very highly personalized process, could potentially morph into no more than a highly sophisticated ATM machine, doling out thousands of results, each travelling so fast on the conveyor belt that we have little chance to imbue them with meaning. Fundamental changes are afoot and a simple trim of the sails will not suffice. By clinging to first-generation genetic counseling principles, we have already arrived at the point where we are talking as fast as we can.

\section{REFERENCES}

1. Bailey D, Lewis M, Harris $S$, et al. Design and evaluation of a decision aid for inviting parents to participate in a fragile $X$ newborn screening pilot study. J Genet Couns, in press.

2. Roche MI, Skinner D. How parents search, interpret, and evaluate genetic information obtained from the internet. J Genet Couns 2009;18:119-129.

3. Kessler S. Introduction. In: Kessler S. (ed). Genetic Counseling: Psychological Dimensions. Academic Press: New York, 1979:1-15.

4. Roberts J, Chen C, Uhlmann W, Green R; for the REVEAL Study Group Effectiveness of a condensed protocol for disclosing APOE genotype and providing risk education for Alzheimer disease. Genet Med 2012; e-pub ahead of print12 April 2012; doi:10.1038/gim.2012.37.

5. Albada A, van Dulmen S, Ausems MG, Bensing JM. A pre-visit website with question prompt sheet for counselees facilitates communication in the first consultation for breast cancer genetic counseling: findings from a randomized controlled trial. Genet Med 2012;14:535-542. 